\title{
Hodge Theory and the Art of Paper Folding
}

By

\author{
Michael KAPOVICH* and John J. Millson**
}

\begin{abstract}
Using Hodge theory and $L^{2}$-cohomology we study the singularities and topology of configuration and moduli spaces of polygonal linkages in the 2-sphere. As a consequence we describe the local deformation space of a folded paper cone in $\boldsymbol{R}^{3}$.
\end{abstract}

\section{§1. Introduction}

This is a part of a series of our papers [11], [12], [13], [14] where we study interrelations between members of the following diagram:

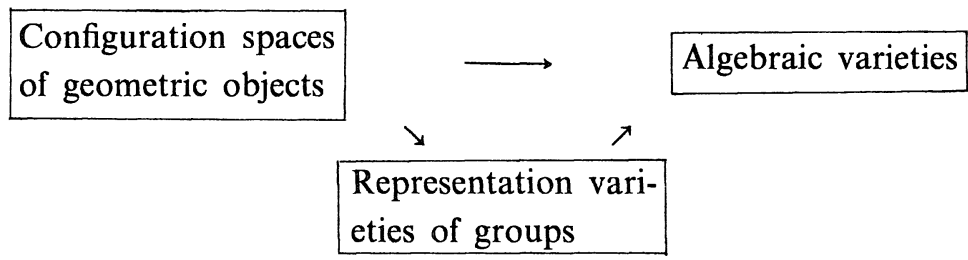

Examples of "geometric objects" that we consider are: linkages in spaces of constant curvature, projective arrangements, folded pieces of paper. Representation varieties under consideration are: representation varieties of infinite Coxeter, Shephard and generalized Artin groups, representation varieties of fundamental groups of hyperbolic 3-manifolds, relative representation varieties of fundamental groups of punctured spheres. The arrows "Configuration spaces" $\rightarrow$ "Algebraic varieties" $\rightarrow$ and "Representation varieties of groups" $\rightarrow$ "Algebraic varieties" are obvious ones, since configuration spaces and

Communicated by Y. Ihara, February 18, 1995. Revised April 16, 1996.

1991 Mathematics Subject Classification(s): 51M09, 32G20

* Department of Mathematics, University of Utah, Salt Lake City, UT 84112, U.S.A.

** Department of Mathematics, University of Maryland, College Park, MD 20742, U.S.A.

This research was partially supported by NSF grant DMS-9306140 at University of Utah (Kapovich) and NSF grants DMS-9205154 and DMS-95-04193 at the University of Maryland (Millson). 
representation varieties have natural structures of algebraic varieties. The arrow "Configuration spaces" $\rightarrow$ "Representation varieties of groups" is not obvious at all, it was introduced in [13]. For certain "Geometric objects" (e.g. polygonal linkages in $\boldsymbol{R}^{3}$ and $\boldsymbol{S}^{3}$ ) the resulting algebraic varieties have a complex-analytic structure and in fact coincide with moduli spaces of complex algebraic objects.

Our general goal to to see how properties of "Geometric objects" and "Groups" are reflected in local and global topology of "Algebraic varieties". In [11] we relate configuration spaces of $n$-gon linkages in $\boldsymbol{R}^{3}$, relative representation varieties of the fundamental group of the $n$-times punctured 2 -sphere into the Euclidean group and Mumford quotients of the $n$-fold product $\left(\boldsymbol{C} \boldsymbol{P}^{1}\right)^{n}$ by $\operatorname{PSL}(2, \boldsymbol{C})$.

In [12] while studying the diagram

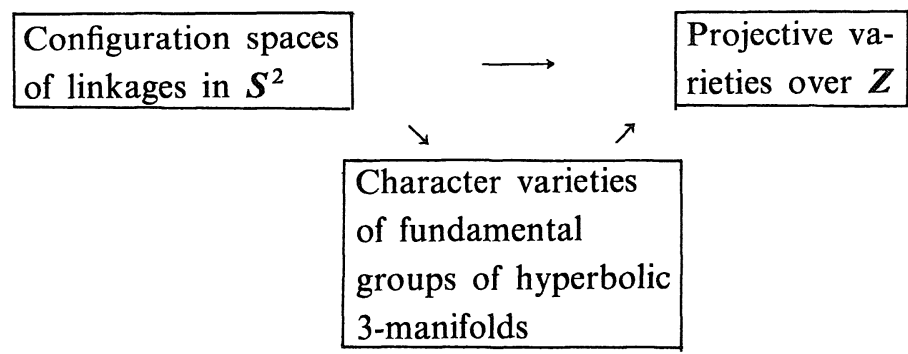

we construct compact hyperbolic 3-manifolds whose representation varieties have nonquadratic singularities. In [14] we consider the diagram

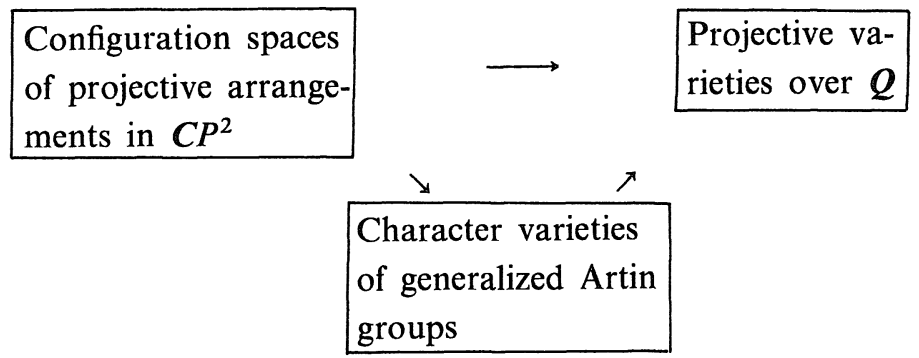

and show that the correspondence "Character varieties of (generalized) Artin groups" $\rightarrow$ "Projective varieties over $Q$ " is essentially onto. In particular, representation varieties of (generalized) Artin groups could have arbitrarily complicated singularities. In [14] we deduce from this that there exist infinitely many Artin groups that are not fundamental groups of smooth complex quasi-projective varieties. 
In this paper the "Geometric objects" are polygonal linkages in $S^{2}$ and the simplest folded pieces of paper in $\boldsymbol{R}^{3}$, "Representation varieties" are relative representation varieties of fundamental groups of punctured 2-spheres. The restriction to polygonal linkages in $\boldsymbol{S}^{2}$ results in restrictions in the global topology and local singularities of the corresponding "Algebraic varieties". Namely, the fact that our groups are fundamental groups of punctured spheres implies that their representation varieties have some extra structure coming from the Hodge theory, which is the main technical tool of the current paper.

Let $\Pi$ be a (marked) geodesic $n$-gon on $S^{2}$ with side-lengths $r$ $=\left(r_{1}, \cdots, r_{n}\right)$. Let $C_{r}$ be the configuration space of $n$-gon linkages with side-lengths $r$ and $M_{r}=C_{r} / S O(3)$ be their moduli space, see $\S 3$. It is immediate that $C_{r}$ and $M_{r}$ are the sets of real points of affine schemes over $R$, also denoted $C_{r}$ and $M_{r}$ respectively. Let [ $\left.\Pi\right]$ be the image of $\Pi$ in $M_{r}$. Our first theorem determines the local nature of $C_{r}$ and $M_{r}$.

Define an $n$-gon linkage $\Pi$ to be degenerate if it lies in a great circle $S^{1}$ of $S^{2}$. Suppose $\Pi$ is degenerate. We orient $S^{1}$ and define $\varepsilon_{i} \in\{ \pm 1\}$ to be 1 if the orientation of the $i$-th edge agrees with that $S^{1}$ and -1 otherwise. We say that the $i$-th edge of $\Pi$ is a forward-track if $\varepsilon_{i}=1$ and a back-track otherwise. Let $f=f(\Pi)$ be the number of forward-tracks and $b=b(\Pi)$ be the number of back-tracks, so $f+b=n$. Define the winding number $w=w(\Pi)$ by

$$
\sum_{i=1}^{n} \varepsilon_{i} r_{i}=2 \pi w
$$

Then we have

Theorem 1.1. (i) $\operatorname{dim} C_{r}=n, \operatorname{dim} M_{r}=n-3$, where dimension is the Krull dimension.

(ii) If $\Pi$ is nondegenerate then $C_{r}$ is smooth at $\Pi$ and $M_{r}$ is smooth at [П].

(iii) If $\Pi$ is degenerate with $f, b, w$ as above, then the germ of $C_{r}$ at $\Pi$ is analytically isomorphic to the germ of $Z(Q)$ at 0 , where $Q$ is a quadratic form on $\boldsymbol{R}^{n+1}$ of nullity 3 and signature $(f-2 w-1, b+2 w-1)$, and $Z(Q)$ denotes the null-cone of $Q$.

(iv) If $\Pi$ is as in (iii) then the germ of $M_{r}$ at [П] is analytically isomorphic to the germ of $Z\left(Q^{\prime}\right)$ at 0 , where $Q^{\prime}$ is a nondegenerate quadratic form on $R^{n-2}$ of signature $(f-2 w-1, b+2 w-1)$.

As a corollary we determine the (locally) rigid spherical $n$-gon linkages. 
Corollary 1.2. A linkage in $S^{2}$ is rigid if and only if it is degenerate and either $f=2 w+1$ or $b=-2 w+1$.

Remark 1.3. If the perimeter $r_{1}+\cdots+r_{n} \leq 2 \pi$, then the rigidity of the linkages above is obvious. If $w \neq 0$ only one of the two equations above is possible (depending of whether or not $w>0, w<0$ ) and the rigidity of the linkages is less obvious.

We apply Theorem 1.1 to determine the local deformation space of a folded paper cone in the Euclidean 3-space. A mathematical model for a folded piece of paper is a pair consisting of a graph $Y$ in the Euclidean plane $E^{2}$ such that the edges of $Y$ are line segments (possibly infinite or half-infinite) and a continuous map $f: E^{2} \rightarrow E^{3}$ such that the restriction of $f$ to each component of $E^{2}-Y$ is a totally-geodesic isometric embedding. Thus we introduce dihedral angles along the edges of $Y$ in such a way that the cone angles around all vertices remain equal to $2 \pi$. Our mathematical model allows the piece of paper to intersect or overlap itself. We let $f_{0}: \mathbb{E}^{2} \rightarrow \mathbb{E}^{3}$ be the totally-geodesic isometric inclusion (the unfolded piece of paper). Note that we do not divide out the space of paper-foldings by the action of the group of isometries $E(3)$ of the Euclidean space. In this paper we determine the local analytic structure of $C(Y)$ in the basic case when $Y$ consists of $n \geq 3$ rays emanating from a single vertex. We assume the angles between adjacent rays are all less than $\pi$.

We prove that $C(Y)$ is quadratic at $f_{0}$. More precisely we prove the following theorem

Theorem 1.4. Assume that all angles between adjacent rays are less than $\pi$. Then there is a neighborhood of $f_{0}$ in $C(Y)$ which is real analytically equivalent to $U \times V$ where $U$ is an open ball in $\boldsymbol{R}^{6}$ and $V$ is a neighborhood of $O$ in the quadratic cone 2 in $\boldsymbol{R}^{n-2}$ given by

$$
\mathscr{Q}=\left\{\left(x_{1}, \cdots, x_{n-2}\right) \in \boldsymbol{R}^{n-2}: x_{1}^{2}+\cdots+x_{n-3}^{2}-x_{n-2}^{2}=0\right\}
$$

Our final theorem gives a "wall-crossing" algorithm to determine the topology of the moduli spaces $M_{r}$. Our results depend on a result of Galitzer [6] describing the set $D_{n}\left(S^{2}\right)$ of $n$-tuples $r=\left(r_{1}, \cdots, r_{n}\right)$ which are side-lengths of a closed $n$-gon in $S^{2}$. It turns out that $D_{n}\left(S^{2}\right)$ is a convex polyhedron which is discussed in $\S 8$. Let $\mathscr{Q}_{n}$ be the space of all $n$-gons in $S^{2}$ up to the action 
of $S O(3)$. We then have a map $\pi: \mathscr{Q}_{n} \rightarrow D_{n}\left(S^{2}\right)$ which assigns to an $n$-gon $\sigma$ its side-lengths $r=\left(r_{1}, \cdots, r_{n}\right)$. Thus the moduli spaces $M_{r}$ are the fibers $\pi^{-1}(r)$. The set of critical values of $\pi$ inside $D_{n}\left(S^{2}\right)$ is a union of hyperplane sections of $D_{n}\left(S^{2}\right)$ called walls. The connected components of the complement of the union of walls in $D_{n}\left(S^{2}\right)$ are called chambers. Since $\pi$ is proper the moduli spaces $M_{r}$ are all diffeomorphic if $r$ varies within a chamber. It is easy to determine $M_{r}$ for special values of $r$ (e.g. if the perimeter less than $2 \pi$ and one side is much larger than the other sides, then $M_{r} \cong S^{n-3}$, c.f. [10]). Thus, if we can determine how $M_{r}$ changes when we cross a wall, then we can compute the topology of $M_{r}$ (though in practice formidable combinatorial problems occur).

First we give Galitzer's description of the walls. For each subset $I \subset\{1, \cdots, n\}$ we let $\bar{I}$ be the complement of $I,|I|$ be the cardinality of $I$ and denotes $\Sigma_{i \in I} r_{i}$. If $w$ is a nonnegative integer we let $H_{I, w}$ be the hyperplane in $\boldsymbol{R}^{n}$ given by

$$
r_{I}-r_{\bar{I}}=2 \pi w
$$

Then Galitzer proves $H_{I . w} \cap D_{n}\left(S^{2}\right)^{0} \neq \emptyset$ if and only if $|I| \geq 2 w+2$. Moreover, all walls of $D_{n}\left(S^{2}\right)$ are of this form. We now state our wall-crossing formula, we will give a slightly more general version in Theorem 8.10.

Theorem 1.5. Let $L$ be an oriented line segment in $D_{n}\left(S^{2}\right)$ crossing the wall $H_{I, w}$ transversally at $r^{*}$ and not meeting any other wall. Let $u^{*}$ be a degenerate linkage with $\pi\left(u^{*}\right)=r^{*}$. Let $r^{\prime}, r^{\prime \prime} \in L$ be the end-points. Then $M_{r^{\prime \prime}}$ is obtained from $M_{r^{\prime}}$ by surgery of the type:

$$
(|I|-2 w-1, n-|I|+2 w-1) \quad \text { or } \quad(n-|I|+2 w-1,|I|-2 w-1)
$$

(depending on the orientation of $L$ and the orientation of the great circle containing $\left.u^{*}\right)$.

Remark 1.6. It is interesting to note that in our proof (see Lemma 8.8 and $\S 8$ for definitions and notation) the deformation theory of representations (the cupproduct $Q$ ) determines the Morse theory (the Hessian $Q_{L}^{*}=d^{2}\left(r_{n} \mid X_{L}\right)_{u^{*}}$ ).

If we take polygonal linkages in $\boldsymbol{S}^{3}$ (instead of $\boldsymbol{S}^{2}$ ) as our "Geometric objects", then the resulting "Algebraic varieties" have a complex-analytic structure - they are the moduli spaces of rank 2 parabolically stable bundles over $\boldsymbol{S}^{2}$ of Mehta and Seshadri [16]. The equivalence is obtained as follows. By $[5, \S 4$, page 129$]$ (see also [15, Lemma 2.7]), the moduli space 
of an $n$-gon linkage in $S^{3}$ is isomorphic to the relative representation variety $\operatorname{Hom}(\Gamma, S ; S U(2)) / S U(2)$, where $\Gamma$ and $S$ are as in $\S 5$ of our paper (in particular, $\Gamma$ is the fundamental group of the $n$ times punctured 2-sphere). By Simpson's generalization [18] of [16] to the genus zero case, the above relative representation variety is isomorphic to the moduli space of parabolically stable bundles over $\boldsymbol{S}^{2}$.

In this case our Hodge theory implies that the singularities are complex-analytically isomorphic to the quadratic cones of [11, Lemma 2.5]. It is easy to see that $D_{n}\left(S^{m}\right)=D_{n}\left(S^{2}\right)$ (here $D_{n}\left(S^{m}\right)$ is the set of possibe side-lengths of closed geodesic $n$-gons in $S^{m}$ ). Accordingly, the result of Galitzer above is equivalent to Proposition 4.4 of [2]. As in the case of $S^{2}$ the deformation theory determines the Morse theory and we obtain the "wall-crossing" formula of [1, Theorem 3.1]. Similar results are also contained in [15]. Thus the algebraic varieties associated to the $n$-gon linkages in $\boldsymbol{R}^{\mathbf{3}}$ and $S^{\mathbf{3}}$ have a description as moduli spaces in algebraic geometry. Is there such an algebraic description for the case of $n$-gon linkages in hyperbolic 3-space?

We would like to thank Dick Hain and Mark Stern for help with the $L^{2}$-cohomology calculations of this paper and Amy Galitzer for allowing us to include results from her $\mathrm{PhD}$ thesis. We would also like to thank Robert Bryant for suggestions that led to the "wall-crossing" approach used here and in [10]. Finally we thank Dick Hain for suggesting the title of this paper.

\section{§2. Relative Deformation Theory}

In this section we review the material of [13]. By the relative deformation theory of a representation $\rho_{0}$ we mean the following. Let $\Gamma$ be a finitely generated group, $G$ be the group of real points of an algebraic group over $\boldsymbol{R}$ which will also be denoted by $G$ and $S=\left\{\Gamma_{1}, \cdots, \Gamma_{n}\right\}$ a collection subgroups of $\Gamma$. We denote by $\mathscr{G}$ the Lie algebra of $G$. Let $\rho_{0}: \Gamma \rightarrow G$ be a homomorphism such that the $A d G$-orbits of $\left.\rho_{0}\right|_{\Gamma_{j}}$ in $\operatorname{Hom}(\Gamma, G)$ are closed, $1 \leq j \leq n$. We then define the space of relative deformations of $\rho_{0}$, to be denoted by $\operatorname{Hom}(\Gamma, S ; G)$, to be the affine subvariety of $\operatorname{Hom}(\Gamma, G)$ consisting of those $\rho$ such that $\left.\rho\right|_{\Gamma_{j}}$ is in the $A d G$ orbit of $\left.\rho_{0}\right|_{\Gamma_{j}}$.

Assume that we have realized $\Gamma$ as the fundamental group of a smooth connected manifold $M$ (possibly with boundary) containing disjoint domains $U_{1}, \cdots, U_{n}$ such that $\Gamma_{j}$ is the image of $\pi_{1}\left(U_{j}\right)$ in $\Gamma$ after suitable choice of base-points and approach paths. Let $H$ be a finite group of diffeomorphisms

acting effectively on $M$ so that each domain $U_{j}$ is invariant under this 
action. We set $U=\cup_{j=1}^{n} U_{j}$ and let $P$ be the flat principal $G$-bundle over $M$ associated to $\rho_{0}$. Let $a d P$ be the associated bundle of Lie algebras. We construct a controlling differential graded Lie algebra $B^{*}(M, U ; a d P)$ of $a d P$-valued differential forms on $M$ for the relative deformations of $\rho_{0}$. Roughly speaking this means we can calculate the deformation space of $\rho_{0}$ by solving the integrability equation

$$
d \eta+\frac{1}{2}[\eta, \eta]=0
$$

in $B^{*}(M, U ; a d P)$. Precisely this means that the complete local $R$-algebra $R_{B_{0}^{*}}$ associated to $B_{0}^{*}$ by the procedure of [17] is isomorphic to the complete local ring of the real-analytic germ $\left(\operatorname{Hom}(\Gamma, S ; G), \rho_{0}\right)$. We define $B^{0}(M, U ; a d P)$ to be the subalgebra of smooth sections of $a d P$ whose restrictions to $U_{j}$ are parallel, $1 \leq j \leq m$. For $i>0$ we define $B^{i}(M, U ; a d P)$ to be the subspace of smooth adP-valued forms that vanish on $U_{j}, 1 \leq j \leq n$. We define an augmentation

$$
\varepsilon: B^{\bullet}(M, U ; a d P) \rightarrow \mathscr{G}
$$

as follows. Let $m \in M$ be a base-point chosen so that $m \notin U$. We define

$$
\varepsilon: B^{0}(M, U ; a d P) \rightarrow \mathscr{G}
$$

by evaluation at $m$ and $\varepsilon: B^{i}(M, U ; a d P) \rightarrow \mathscr{G}$ to be zero if $i>0$. We let $B^{*}(M, U ; a d P)_{0}$ be the augmentation ideal of $B^{*}(M, U ; a d P)$, i.e. the kernel of $\varepsilon$. Then $B^{*}(M, U ; a d P)_{0}$ is a controlling differential graded Lie algebra for the relative deformations of $\rho_{0}$ by Theorem 2.9 of [13].

Remark 2.1. Let $E$ be any flat bundle over $M$. Then we may define a complex $B^{*}(M, U ; E)$ by replacing $a d P$ in the above definition by $E$. We will use this notation throughout this paper without further comment.

We have an extension of groups

$$
1 \rightarrow \Gamma \rightarrow \Phi \rightarrow H \rightarrow 1
$$

where $\Phi$ is the orbifold fundamental group of $M / H$. We assume that $\rho_{0}$ extends to $\Phi$, we retain the notation $\rho_{0}$ for the extension. Let $\Phi_{j}, 1 \leq j \leq n$, be the orbifold fundamental groups of $U_{j} / H$ and define $R=\left\{\Phi_{1}, \cdots, \Phi_{n}\right\}$. By Theorem 2.10 of [13], the algebra of invariants $B^{*}(M, U ; a d P)_{0}^{H}$ controls the germ 
$\left(\operatorname{Hom}(\Phi, R ; G) ; \rho_{0}\right)$.

We will need the following general result about controlling differential graded Lie algebras.

Definition 2.2. A subvariety $S \subset \operatorname{Hom}(\Phi, R ; G)$ is said to be a cross-section to the orbits of $G$ if the map $\phi: G \times S \rightarrow \operatorname{Hom}(\Phi, R ; G)$ given by $\phi(g, s)=A d(g) s$ is an isomorphism of varieties.

Definition 2.3. Suppose there exists a G-invariant open neighborhood $V$ of $\rho_{0}$ and an analytic subvariety $S$ of $V$ such that $\rho_{0} \in S$ and the natural map $\phi: G \times S \rightarrow V$ is an isomorphism of analytic spaces. Then we call $S$ a local cross-section through $\rho_{0}$ to the orbits of $G$.

Theorem 2.4. Suppose a local cross-section through $\rho_{0}$ exists. Then the algebra of invariants $B^{\circ}(M, U ; a d P)^{H}$ controls the germ $\left(\operatorname{Hom}(\Phi, R ; G) / G ;\left[\rho_{0}\right]\right)$.

Proof. We let $S$ be the local cross-section and $V=G S$. Clearly the germs

$$
\left(S, \rho_{0}\right) \quad \text { and } \quad\left(\operatorname{Hom}(\Phi, R ; G) / G ;\left[\rho_{0}\right]\right)
$$

are isomorphic. We now prove that $B^{\circ}(M, U ; a d P)^{H}$ controls the germ $\left(S, \rho_{0}\right)$. We will use the notations of $\S 2$ from [13] freely.

If $A$ is an Artin local $R$-algebra and $\left(X, x_{0}\right)$ is an analytic germ we will use $X_{A}^{0}$ to denote the set of $A$-points of $\left(X, x_{0}\right)$. We note that $G_{A}^{0}=\exp (\mathscr{G} \otimes \mathscr{M})$ where $\mathscr{M}$ is the maximal ideal of $A$. We will abbreviate $\operatorname{Hom}(\Phi, R ; G)$ to $X$ and $B^{\circ}(M, U ; a d P)^{H}$ to $L^{\circ}$. We have an isomorphism (of functors of $A$ )

$$
V_{A}^{0}=X_{A}^{0} \cong G_{A}^{0} \times S_{A}^{0}
$$

Let $\left(P, \omega_{0}\right)$ be the flat principal bundle over $M$ associated to $\rho_{0}$. The assignment of the holonomy representation to a flat connection induces the functor

$$
\text { hol: } \mathscr{F}_{A}^{r}\left(\omega_{0}\right) \rightarrow X_{A}^{0}
$$

of $[13, \S 2]$. We let $\mathscr{S}_{A}^{0}$ be the inverse image of $S_{A}^{0}$ under hol and $G^{0}\left(P_{A}\right)$ be the deformed gauge group of $[13, \S 2]$. We assume we have chosen $p \in P$. In [13] we defined

$$
\varepsilon_{p}: G^{0}\left(P_{A}\right) \rightarrow G_{A}^{0}
$$


by $F(p)=p \varepsilon_{p}(F)$. Then $\operatorname{hol}\left(F^{*} \omega\right)=\varepsilon_{p}(F) h o l(\omega)$. It is immediate that the above product decomposition induces a decomposition

$$
\mathscr{F}_{A}^{r}\left(\omega_{0}\right) \cong G^{0}\left(P_{A}\right) \times_{\mathrm{ker} \varepsilon_{p}} \mathscr{S}_{A}^{0}
$$

We recall that the complete local ring $R_{L}$ is a hull for the functor $I \operatorname{so} \mathscr{C}(L, A)$. By Proposition 2.7 of [13] the functor $I s O \mathscr{C}(L, A)$ is isomorphic to $\mathscr{F}_{A}^{r}\left(\omega_{0}\right) / \boldsymbol{G}^{0}\left(P_{A}\right)$. But clearly we have isomorphisms (natural in $A$ ):

$$
\mathscr{F}_{A}^{r}\left(\omega_{0}\right) / G^{0}\left(P_{A}\right) \cong \mathscr{S}_{A}^{0} / \operatorname{ker} \varepsilon_{p} \cong S_{A}^{0}
$$

Hence $\hat{\mathcal{O}}_{S, \rho_{0}}$ pro-represents $I s o \mathscr{C}(L, A)$ and consequently is a hull for Iso $\mathscr{C}(L, A)$. Since hulls are unique we have $\hat{\mathcal{C}}_{S, \rho_{0}} \cong R_{L}$.

\section{§3. Configuration Spaces of Spherical Polygonal Linkages}

In this section we will begin our study of the configuration spaces of polygonal linkages $C_{r}$ and moduli spaces $M_{r}$. Here $r=\left(r_{1}, \cdots, r_{n}\right)$ is an element of the $n$-fold Cartesian product $I^{n}$, where $I=(0, \pi)$. We now give necessary definitions.

Definition 3.1. An n-gon $\pi=\left(\sigma_{1}, \cdots, \sigma_{n}\right)$ is an $n$-tuple of oriented geodesic arcs $\sigma_{j}$ (in $\boldsymbol{S}^{2}$ ) of lengths between 0 and $\pi$ (inclusive) such that the end-point of $\sigma_{i-1}$ is equal to the initial point of $\sigma_{i}, 1 \leq i \leq n+1$ (the indices are taken modulo $n$ ).

Definition 3.2. $A$ free linkage with $n$ vertices is an $n$-tuple of oriented geodesic arcs $\sigma_{j}\left(\right.$ in $S^{2}$ ) of lengths between 0 and $\pi$ such that the end-point of $\sigma_{i-1}$ is equal to the initial point of $\sigma_{i} 1 \leq i \leq n$.

We let $r_{i}$ be the length of $\sigma_{i}$ in the spherical metric. The $\operatorname{arcs} \sigma_{1}, \cdots, \sigma_{n}$ will be called the edges of $\Pi$. We will use $u=\left(u_{1}, \cdots, u_{n}\right)$ to denote the set of vertices of $\Pi$, that is the set of initial points of the edges $\sigma_{i}$. In case $0 \leq r_{i}<\pi$ the polygon $\Pi$ is determined by its vertex set $u$ and we will write $\Pi=\left(u_{1}, \cdots, u_{n}\right)$. We will sometimes write $u$ instead of $\Pi$.

Definition 3.3. Let $r \in I^{n}$. The configuration space $C_{r}$ of (marked) n-gon linkages on $\boldsymbol{S}^{2}$ with side-lengths $r=\left(r_{1}, \cdots, r_{n}\right)$ is the set of all $n$-gons $u=\left(u_{1}, \cdots, u_{n}\right)$ such that the distances $d\left(u_{i}, u_{i+1}\right)$ in the spherical metric satisfy $d\left(u_{i}, u_{i+1}\right)=r_{i}$, $1 \leq i \leq n$. 
It is immediate that $C_{r}$ is the set of real points of the affine scheme over $\boldsymbol{R}$ defined by

$$
\left(u_{i}, u_{i+1}\right)=\cos r_{i}, \quad 1 \leq i \leq n
$$

where $(\cdot, \cdot)$ denotes the scalar product in $R^{3}$. The group $S O(3)$ acts on $C_{r}$ according to

$$
g \cdot u=\left(g u_{1}, \cdots, g u_{n}\right), u \in C_{r}, g \in S O(3)
$$

Definition 3.4. The moduli space $M_{r}$ of $n$-gon linkages on $S^{2}$ with side-lengths $r=\left(r_{1}, \cdots, r_{n}\right)$ is defined to be the quotient scheme of $C_{r}$ by $S O(3)$.

In fact there are no difficulties in passing to the quotient in this case because there exists a cross-section $S_{r}$ to the $S O(3)$-orbits in $C_{r}$. Indeed, we define $S_{r}$ to be the subvariety of $C_{r}$ such that $u_{1}$ coincides with the first standard basis vector $\vec{e}_{1}$ of $\mathbb{R}^{3}$ and $u_{2}$ lies on the half-equator in $S^{2}$ defined by

$$
\left(\vec{e}_{3}, u_{2}\right)=0, \quad \text { and } \quad\left(\vec{e}_{2}, u_{2}\right)>0
$$

Lemma 3.5. The variety $S_{r}$ is a cross-section to the $S O(3)$-orbits in $C_{r}$.

Proof. It is obvious that $S_{r}$ is a set-theoretic cross-section. To see that $S_{r}$ is a scheme-theoretic cross-section we embed $C_{r}$ into $\tilde{C}_{r}$, the configuration space of the linkage consisting of $n$ vertices $u_{1}, \cdots, u_{n}$ and a single edge of length $r_{1}$ joining $u_{1}$ and $u_{2}$. We let $\tilde{S}_{r}$ be the subvariety of $\tilde{C}_{r}$ such that $u_{1}$ and $u_{2}$ are as described above. Clearly $\tilde{C}_{r}=S O(3) \times\left(S^{2}\right)^{n-2}$ so $\tilde{C}_{r} \cong S O(3) \times \tilde{S}_{r}$ as schemes. Now $S_{r}$ is the pull-back of the cross-section $\tilde{S}_{r}$ under the equivariant embedding $C_{r} \rightarrow \tilde{C}_{r}$. The reader will verify that the pull-back of a scheme-theoretic cross-section under an equivariant morphism is a schemetheoretic cross-section.

We may then identify the quotient scheme $M_{r}$ with the subscheme $S_{r} \subset C_{r}$. We will compute the real-analytic germ $\left(C_{r}, \Pi\right)$ of $C_{r}$ at $\Pi=u$ $=\left(u_{1}, \cdots, u_{n}\right)$ for any $r$ and $u$ as above. Our goal is to relate $\left(C_{r}, \Pi\right)$ and a germ of a certain relative representation variety.

Let $\Phi$ be the free product of $n$ copies of $\boldsymbol{Z} / 2, \Phi=\left\langle\tau_{1}\right\rangle * \cdots *\left\langle\tau_{n}\right\rangle$ where $\tau_{j}$ corresponds to the vertex $u_{j}$ of a spherical $n$-gon $\Pi$. Let $\gamma_{j}=\tau_{j+1} \tau_{j}$. Let $\rho=\rho_{\Pi}: \Phi \rightarrow S O(3)$ be the representation which assigns to the generator $\tau_{i}$ the 
rotation $s_{u_{i}}$ of 180 degrees around $u_{i}$. We denote by $\Phi_{i}$ the subgroup of $\Phi$ generated by the involutions $\tau_{i}, \tau_{i+1}$, we put $R=\left\{\Phi_{1}, \cdots, \Phi_{n}\right\}$. Now we pick a particular $n$-gon $\Pi^{*}$ in a configuration space $C_{r}$. Theorem 3.2 of [13] implies the following

Theorem 3.6. The map $\Pi \mapsto \rho_{\Pi}$ gives an analytic isomorphism of germs

$$
\left(C_{r}, \Pi^{*}\right) \cong(\operatorname{Hom}(\Phi, R ; S O(3)), \rho)
$$

Corollary 3.7. The map $\Pi \mapsto \rho_{\Pi}$ induces an analytic isomorphism of germs

$$
\left(M_{r},\left[\Pi^{*}\right]\right) \cong(\operatorname{Hom}(\Phi, R ; S O(3)) / S O(3),[\rho])
$$

Proof. Since the isomorphism in Theorem 3.6 is $S O(3)$-equivariant, it induces an isomorphism of quotient germs.

We say that a spherical polygon $\Pi$ is degenerate if it is contained in a great circle of $S^{2}$. As we shall see, degenerate polygons are precisely the singular points of the configuration spaces $C_{r}$ and moduli spaces $M_{r}$. Let's assume that $\Pi \in S_{r}$ is a degenerate polygon, that it lies in the $x y$-plane and the edge $\sigma_{1}$ of $\Pi$ has counterclockwise orientation.

We recall that we associated numbers $f, b, w$ and to $\varepsilon_{j}, 1 \leq j \leq n$ to $\Pi$ in the Introduction. We set $\theta_{j}=\varepsilon_{j} r_{j}$, whence

$$
\sum_{j=1}^{n} \theta_{j}=2 \pi w
$$

We will see that the numbers $f, b$ and $w$ will determine the singularity of $C_{r}$ at $\Pi$.

Let $\rho:=\rho_{\Pi}$ be representation associated with the polygon $\Pi$. We claim a basis $e_{1}, e_{2}, e_{3}$ for $s o(3)$ can be chosen so that $e_{3}=\left[e_{1}, e_{2}\right], e_{3}$ is fixed under $a d\left(\rho\left(\gamma_{j}\right)\right), 1 \leq j \leq n$ and

$$
\operatorname{Ad}\left(\rho_{0}\left(\gamma_{j}\right)\right) e_{1}=\cos \left(2 \theta_{j}\right) \cdot e_{1}+\sin \left(2 \theta_{j}\right) \cdot e_{2}
$$

Indeed, take $e_{j}$ be the images of the vectors $\vec{e}_{j}$ of the natural basis of $\boldsymbol{R}^{3}$ under the canonical isomorphism ad: $\boldsymbol{R}^{3} \rightarrow$ so(3) which is given by

$$
\operatorname{ad} \xi(\eta)=\xi \times \eta
$$




\section{§4. Spherical Polygons and Paper-folding}

Let $Y \subset \boldsymbol{R}^{2}$ be the union of $n$ geodesic rays emanating from the origin, we shall assume that none of the complementary components $\mathbb{R}^{2}-Y$ contains a half-plane, let $r_{1}, \cdots, r_{n}$ be the angles of the complementary regions. Intersections of the rays in $Y$ with the unit sphere $S^{2}$ centered at the origin determine a collection of points $u_{1}^{0}, \cdots, u_{n}^{0} \in \mathbb{S}^{2}$. These points are the vertices of the degenerate spherical polygon $\Pi_{0}$, with the sidelengths $d\left(u_{i}, u_{i+1}\right)=r_{i}$. If $f: \mathbb{R}^{2} \rightarrow \mathbb{R}^{3}$ is a paper-folding with pleating locus $Y$, then $f$ is uniquely determined by the restriction of $f$ to the components of $Y-\{0\}$. We have assumed that $r_{i}<\pi$ for all $i$. Let $C_{r}$ be the configuration space of $\Pi_{0}$.

Theorem 4.1. $C(Y) \cong C_{r} \times \boldsymbol{R}^{3}$ as algebraic varieties.

Proof. Recall that $u_{i} \in \mathbb{R}^{3}, 1 \leq i \leq n$ are vectors tangent to the rays in $Y$. Any map $f \in C(Y)$, is differentiable along the rays in $Y$, hence we have well-defined vectors

$$
v_{i}=d f_{0}\left(u_{i}\right) \in T_{f(0)}\left(\boldsymbol{R}^{3}\right)
$$

The unit vectors $v_{i}$ satisfy the property:

$$
\left(v_{i}, v_{i+1}\right)=\cos \left(r_{i}\right), 1 \leq i \leq n+1 \quad(\bmod n)
$$

It is clear that ordered collections of unit vectors $v_{i} \in T_{x}\left(\boldsymbol{R}^{3}\right), x \in \boldsymbol{R}^{3}$ which satisfy the above equation correspond bijectively to elements $f \in C(Y)$.

Note however that the same equation (1) defines the variety $C_{r}$ as well. Theorem 4.1 follows.

Remark 4.2. With extra care one can generalize the above theorem to the case when one of the angles $r_{i}$ is equal to $\pi$, but we are not going to discuss this case here.

Our problem of determining $\left(C_{r}, \Pi_{0}\right) \times R^{3} \cong\left(C(Y), f_{0}\right)$ then amounts to computing the real-analytic germ of $C_{r}$ at $\Pi_{0}$ for the case in which $r_{1}+r_{2}+\cdots+r_{n}=2 \pi$ and $\Pi_{0}$ is as above.

\section{§5. A Controlling Differential Graded Lie Algebra for the Deformations of a Spherical Polygonal Linkage}

In this section we will describe controlling differential graded Lie algebras 
for the germs $\left(C_{r}, \Pi\right)$ and $\left(M_{r},[\Pi]\right)$. We let $\Phi$ and $R$ be as in $\S 3$. Observe that the spherical polygon $\Pi$ gives rise to the representation $\rho_{\Pi}=\rho_{0}: \Phi \rightarrow S O(3)$ which sends $\tau_{i}$ to the Cartan involution $s_{u_{i}}$ on $S^{2}$ at $u_{i}$. Now we use [13] to construct a differential graded Lie algebra of forms on the $n$ times punctured 2-sphere that controls $\left(C_{r}, \Pi\right)$.

Let $\Gamma \subset \Phi$ be the subgroup of words of even length in $\tau_{1}, \cdots, \tau_{n}$ and put

$$
\gamma_{1}=\tau_{2} \tau_{1}, \gamma_{2}=\tau_{3} \tau_{2}, \cdots, \gamma_{n}=\tau_{1} \tau_{n}
$$

Then $\Gamma$ is generated by the elements $\gamma_{1}, \cdots, \gamma_{n}$, subject to the single relation

$$
\gamma_{n} \gamma_{n-1} \cdots \gamma_{2} \gamma_{1}=1
$$

Thus $\Gamma$ is the fundamental group of the $n$ times punctured sphere

$$
M=S^{2}-\left\{m_{1}, \cdots, m_{n}\right\}
$$

We observe that exact sequence

$$
1 \rightarrow \Gamma \rightarrow \Phi \rightarrow Z / 2 \rightarrow 1
$$

splits. If we split this sequence by sending the generator $\alpha$ of $Z / 2$ to $\tau_{1}$ then we obtain the following action of $Z / 2$ on $\Gamma$ :

$$
\alpha\left(\gamma_{i}\right)=\tau_{1} \gamma_{i} \tau_{1}=\left(\gamma_{1}^{-1} \cdot \cdots \cdot \gamma_{i-1}^{-1}\right) \gamma_{i}^{-1}\left(\gamma_{i-1} \cdots \cdot \gamma_{1}\right)
$$

We let $P$ be the flat principal $S O(3)$-bundle associated to $\rho_{0} \mid \Gamma$ and $a d P$ be the associated Lie algebra bundle. Take $U_{i}$ be the disjoint punctured disc neighborhoods of $m_{i}, 1 \leq i \leq n$, and put $U=U_{1} \cup \cdots \cup U_{n}$. Then by the discussion in $\S 3$, the differential graded Lie algebra $B^{\circ}(M, U ; a d P)_{0}$ is a controlling differential graded Lie algebra for the deformations of $\rho_{0} \mid \Gamma$ relative to the collection of subgroups $S=\left\{\Gamma_{1}, \cdots, \Gamma_{n}\right\}$, where $\Gamma_{j}$ is the cyclic subgroup generated by $\gamma_{j}$. Note that $\Gamma_{j}=\Phi_{j} \cap \Gamma$.

We now may realize $M$ as the standard round sphere with the points $m_{1}, \cdots, m_{n}$ removed from the equator. The action of $H:=Z / 2$ on the round sphere given by reflection in the equator carries $M$ into itself. We choose the discs $U_{i}$ to be invariant under this action. In particular $H$ acts on $\pi_{1}(M)$ and we may form the semidirect product $\Psi:=H \ltimes \pi_{1}(M)$. By definition $\Psi$ is the orbifold fundamental group of the orbifold $M / H$. The following proposition is central what follows. 
Proposition 5.1. $\Psi$ and $\Phi$ are isomorphic.

Proof. We replace $S^{2}$ by $C \cup\{\infty\}$ and the reflection in the equator by reflection in the $x$-axis. We take $0 \in C$ as the basepoint and $1,2, \cdots, n$ as punctures. We let $\mu_{k}$ be the loop that proceeds from 0 to $k$ in the upper half plane, encircles $k$ once in the counterclockwise direction, then returns to 0 in the upper half plane. Clearly $\mu_{1}, \cdots, \mu_{n}$ generate $\pi_{1}(M)$ subject to the relation $\mu_{1} \cdots \mu_{n}=1$. The reader will verify that

$$
\sigma\left(\mu_{k}\right)=\left(\mu_{1} \cdots \mu_{k-1}\right) \mu_{k}^{-1}\left(\mu_{1} \cdots \mu_{k-1}\right)^{-1}, \quad 1 \leq k \leq n
$$

Thus we obtain the required isomorphism by sending $\mu_{k}$ to $\gamma_{k}^{-1}, 1 \leq k \leq n$, and $\sigma$ to $\alpha$.

Thus we may apply Theorem 2.10 of [13] to deduce the following

Theorem 5.2. The subalgebra of $Z / 2$-invariants $B^{*}(M, U ; a d P)_{0}^{Z / 2}$ is a controlling differential graded Lie algebra for the relative deformations of $\rho_{0}$ in $S O(3)$.

Corollary 5.3. The algebra $B^{\cdot}(M, U ; a d P)_{0}^{Z / 2}$ is a controlling differential graded Lie algebra for the germ $\left(C_{r}, \Pi\right)$.

As a consequence of Theorem 2.4 we derive

Theorem 5.4. The differential graded Lie algebra $B^{*}(M, U ; \text { adP })^{Z / 2}$ controls the germ $\left(M_{r},[\Pi]\right)$.

Proof. We have only to produce a cross-section $S$ to the orbits of $S O(3)$ on $C_{r}$. This cross-section $S=S_{r}$ however was constructed in Lemma 3.5.

We can now determine the singular points of $C_{r}$. Our goal is to prove that non-degenerate polygons are smooth points of $C_{r}$. First recall a general result guaranteeing smoothness of the complete local $\mathrm{k}$-algebra $R_{L}$. We sketch a proof for the convenience of the reader.

Theorem 5.5. Suppose $L^{\bullet}$ is a differential graded Lie algebra over a field $\mathbf{k}$ of characteristic zero. Suppose $H^{2}(L)=\{0\}$. Then $R_{L} \cong \mathbf{k}\left[\left[H^{1}(L)\right]\right]$ is smooth. 
Proof. Let $\mathscr{M}_{H^{2}}$ be the maximal ideal corresponding to $\{0\} \in H^{2}(L)$. By Theorem 3.9 of [9], there is a formal map (the Kuranishi map) $F: H^{1}(L) \rightarrow H^{2}(L)$ such that $R_{L}$ is isomorphic to the complete local $\mathrm{k}$-algebra

$$
\mathbf{k}\left[\left[H^{1}(L)\right]\right] / F^{*} \mathscr{M}_{H^{2}(L)}
$$

We can now prove our smoothness theorem for points in $C_{r}$.

Theorem 5.6. If $\Pi$ is nondegenerate then $C_{r}$ is smooth at $\Pi$.

Proof. By Artin's Theorem, see Theorem 3.1 of [8], it suffices to prove that the complete local ring of $C_{r}$ at $\pi$ is isomorphic to $R\left[\left[H^{1}(L)\right]\right]$, where $L=B^{*}(M, U ; a d P)_{0}^{\mathbf{Z} / 2}$. Thus by the previous theorem it suffices to show that $H^{2}(L)=0$. But

$$
H^{2}\left(B^{*}(M, U ; a d P)_{0}^{\mathbf{Z} / 2}\right)=H^{2}\left(B^{*}(M, U ; a d P)^{\mathbf{Z} / 2}\right)
$$

since the differential graded Lie algebras involved differ only in degree zero. By Lemma 2.16 of [13] we have

$$
H^{2}\left(B^{*}(M, U ; a d P)^{\mathbf{Z} / 2}\right) \cong H^{0}(\Phi, s o(3) \otimes \varepsilon)
$$

Here $s o(3)$ is the Lie algebra of $S O(3)$ and $\varepsilon: \Phi \rightarrow\{ \pm 1\}$ is the signum character defined by $\varepsilon\left(\tau_{i}\right)=-1,1 \leq i \leq n$. But

$$
H^{0}(\Phi, s o(3) \otimes \varepsilon) \cong H^{0}(\Gamma, s o(3))^{\varepsilon}
$$

where the $\varepsilon$ denotes the $\varepsilon$-isotypic subspace for the induced action of the group $Z / 2$ on $H^{0}(\Gamma, s o(3))$. If $\Pi$ is nondegenerate the action of $\Gamma$ is irreducible and $H^{0}(\Gamma, s o(3))=\{0\}$.

Thus we will assume henceforth that $\Pi$ is degenerate and is contained in the equator of $S^{2}$ and equivalently that the image $\rho_{0}(\Gamma)$ is contained in $S O(2)$ (which we identify with the subgroup of $S O(3)$ fixing the third coordinate vector of $\boldsymbol{R}^{3}$ ). Thus we have a decomposition

$$
a d P=E_{0} \oplus E
$$

where $E_{0}$ is the trivial one-dimensional flat bundle and $E$ is the 2-dimensional 
flat bundle corresponding to an irreducible representation from $\Gamma$ into $S O(2)$. Of course we have

$$
E \otimes C=L \oplus L^{\vee}
$$

where $L$ is a flat complex line bundle and $L^{v}$ is its dual.

We conclude this section with some remarks on the cup-product (or bracket)

$$
Q: H^{1}\left(B^{\circ}(M, U ; a d P)\right) \rightarrow H^{2}\left(B^{\circ}(M, U ; a d P)\right)
$$

\section{Lemma 5.7.}

$$
H^{2}\left(B^{\circ}(M, U ; a d P)\right) \cong \boldsymbol{R}
$$

Proof. We write $a d P=E_{0} \oplus E$ as above and observe by Poincare duality that

$$
\begin{aligned}
& H^{2}\left(B^{\circ}(M, U ; E)\right) \cong H^{0}(M, E)=\{0\} \\
& H^{2}\left(B^{\circ}\left(M, U ; E_{0}\right)\right) \cong H^{0}\left(M, E_{0}\right)=\boldsymbol{R}
\end{aligned}
$$

Thus $Q$ is a scalar-valued quadratic form. We observe that $Q$ is induced by the tensorial bilinear form

$$
b: T^{*}(M) \otimes a d P \times T^{*}(M) \otimes a d P \rightarrow \Lambda^{2} T^{*}(M) \otimes a d P
$$

given by $b=b_{1} \otimes b_{2}$ where $b_{1}$ is the wedge product and $b_{2}$ is the Lie bracket.

\section{§6. Formality Via $L^{2}$-cohomology and Hodge Theory}

We now give $M$ a complete hyperbolic metric and let

$$
A_{(2)}^{\circ}(M, a d P)
$$

be the differential graded Lie algebra of smooth $a d P$-valued forms $\eta$ on $M$ such that $\eta$ and $d \eta$ are square integrable for the hyperbolic metric on $M$ and the parallel metric on $a d P$. Since $M$ has finite area we have an inclusion

$$
j: B^{\circ}(M, U ; a d P) \rightarrow \underset{A_{(2)}^{*}(M, a d P)}{ }
$$

We then have the following theorem. 
Theorem 6.1. The inclusion $j$ is a quasi-isomorphism.

Proof. The proof of the theorem will be contained in the next three lemmas. We let $i: M \rightarrow C \boldsymbol{P}^{1}$ be the inclusion. It is easy to see that there is a cover of $C \boldsymbol{P}^{1}$ by convex open sets $V_{i}, 1 \leq i \leq N$, such that each puncture $m_{i}$ is contained in at most one of the $V_{i}$ 's. Furthermore, there is a partition of unity $\left\{\varphi_{i}, 1 \leq i \leq N\right\}$ subordinate to the $V_{i}$ 's and such that $\varphi_{i}$ is constant in a neighborhood $U_{j}$ of each $m_{j}, 1 \leq j \leq n$.

Now let $\mathscr{B}^{\circ}$ and $\mathscr{A}_{(2)}^{\circ}$ denote the complexes of sheaves on $M$ corresponding to $B^{\circ}(M, U ; a d P)$ and $A_{(2)}^{\circ}(M, a d P)$ respectively. We obtain the corresponding "push-forward" complexes $i_{*} \mathscr{B}^{\circ}$ and $i_{*} \mathscr{A}_{(2)}^{\circ}$ on $\boldsymbol{C P}^{1}$. Then it is immediate that for any open subset $D \subset C \boldsymbol{P}^{1}$ spaces $i_{*} \mathscr{B}^{\circ}(D)$ and $i_{*} \mathscr{A}_{(2)}^{*}(D)$ are closed under multiplication by $\varphi_{j}, 1 \leq j \leq N$. Thus we have the following lemma.

Lemma 6.2. The complexes of sheaves $\mathscr{B}^{\circ}$ and $\mathscr{A}_{(2)}^{\circ}$ are complexes of fine sheaves.

We let $\overparen{a d P}$ denote the locally constant sheaf associated to the flat bundle $a d P$. The following lemma is obvious.

Lemma 6.3. The inclusion

$$
i_{*} \widetilde{a d P} \subseteq i_{*} \mathscr{B}^{\circ}
$$

is a quasi-isomorphism of complexes of sheaves.

The next lemma is not obvious but follows from [19].

Lemma 6.4. The inclusion

$$
i_{*} \widetilde{a d P} \varsigma i_{*} \mathscr{A}_{(2)}^{.}
$$

is a quasi-isomorphism of complexes of sheaves.

Proof. We recall that $a d P=E_{0} \oplus E$ where $E_{0}$ is a trivial one-dimensional uinitary local system and $E$ is irreducible. Then the above inclusion is a direct sum of two inclusions. The inclusion corresponding to $E$ is a quasi-isomorphism by [4, Theorem D2]. The inclusion corresponding to $E_{0}$ is a quasi-isomorphism by [19, Proposition 6.6]. 
Theorem 6.1 now follows by the standard double complex argument, see [7, Theorem 4.6.6].

Theorem 6.1 has a large number of consequences which we now enumerate.

Theorem 6.5. The augmented differential graded Lie algebra $B^{\circ}(M, U ; a d P)$ is formal.

Proof. By Theorem 6.1 it suffices to prove that ${\dot{A_{(2)}}}^{\circ}(M, a d P)$ is formal. But by Theorem 2.7 of [19] we have the expected relations among the Laplacians associated to $\partial, \bar{\partial}$ and $d$, namely

$$
\Delta_{\partial}=\Delta_{\bar{\partial}}=\frac{1}{2} \Delta_{d}
$$

The $d d^{c}$-lemma follows by the usual argument, [3, Lemma 5.11]. We then obtain the standard quasi-isomorphism

$$
\left(B^{\bullet}(M, U ; a d P)\right) \leftarrow\left(\operatorname{Ker} d^{c}, d\right) \rightarrow\left(\operatorname{Ker} d^{c} / \operatorname{Im} d^{c}, 0\right)
$$

The details may be found in [8, Section 7].

In fact we need the following consequences of the above theorem.

Theorem 6.6. The augmented differential graded Lie algebra of $Z / 2$ invariants

$$
B^{\bullet}(M, U ; a d P)^{\mathbf{Z} / 2}
$$

is formal.

Proof. The above quasi-isomorphism induces a quasi-isomorphism of $Z / 2$-invariants.

Corollary 6.7. The germ of the relative representation variety

$$
\left.\operatorname{Hom}(\Phi, R ; S O(3)), \rho_{0}\right)
$$

is quadratic for the representation $\rho_{0}: \Phi \rightarrow S O(3)$ associated with any degenerate polygon $\Pi_{0}$.

Proof. The corollary follows from [8, Theorem 3.5]. 
Corollary 6.8. The germ $\left(C_{r}, \Pi_{0}\right)$ is quadratic for any degenerate polygon $\Pi_{0}$. The corollary follows from Theorem 6.6 and [8, Corollary 3.6].

Corollary 6.9. The germ $\left(M_{r},\left[\Pi_{0}\right]\right)$ is quadratic for any degenerate polygon $\Pi_{0}$.

Proof. The corollary follows from Theorem 6.6 and [8, Corollary 3.6.].

It remains to compute the quadratic equations defining $\left(C_{r}, \Pi\right)$ and $\left(M_{r},\left[\Pi_{0}\right]\right)$. Thus we must compute the cup product

$$
Q: H^{1}\left(B^{\bullet}(M, U ; a d P)\right) \otimes H^{1}\left(B^{*}(M, U ; a d P)\right) \rightarrow H^{2}\left(B^{*}(M, U ; a d P)\right)
$$

This will be done in the next section.

\section{§7. Calculation of the Cup Product from the Hodge-Riemann Bilinear Relations}

In what follows we will use the notation $H_{(2)}^{*}(M, a d P)$ for the cohomology of the complex $A_{(2)}^{\circ}(M, a d P)$. Thus we have as a consequence of Theorem 6.1 a natural isomorphism

$$
H(j): H^{\bullet}\left(B^{\bullet}(M, U ; a d P) \rightarrow H_{(2)}^{\bullet}(M, a d P)\right.
$$

Before stating our final consequence of Theorem 6.1 we need a definition.

Definition 7.1. $A$ Hodge complex is a pair of complexes $A^{*}=\left(A_{R}^{*},\left(A_{C}^{*}, F\right)\right)$ and a quasi-isomorphism $A_{\boldsymbol{R}}^{\dot{2}} \otimes C \rightarrow A_{\boldsymbol{C}}^{*}$ such that:

(1) $H^{k}\left(A_{\boldsymbol{R}}^{*}\right)$ is finite dimensional for all $k$.

(2) $F^{*}$ is a decreasing filtration of $\dot{A}_{C}^{*}$ of finite length.

(3) The differential $d$ of $A_{C}^{*}$ is strict with respect to $F^{*}$ (i.e. an element of $F^{p} A_{\boldsymbol{C}}^{*}$ is exact in $A_{\boldsymbol{C}}^{*}$ if and only if it is exact in $F^{p} A_{\boldsymbol{C}}^{*}$ ).

(4) $\left(H^{k}\left(A_{\boldsymbol{R}}^{*}\right), H^{k}\left(A_{\boldsymbol{C}}^{*}\right), F^{*}\right)$ is a Hodge structure of weight $k$ (see $\left.[3, \S 5.19]\right)$.

We define a filtration $F^{\cdot}$ on $A_{(2)}^{\bullet}\left(M, a d P_{C}\right)$ as follows.

(i) $F^{0} \hat{A}_{(2)}\left(M, a d P_{C}\right)=\dot{A_{(2)}^{*}}\left(M, a d P_{C}\right)$,

(ii) $F^{1} A_{(2)}^{0}\left(M, a d P_{c}\right)=A_{(2)}^{1,0}\left(M, a d P_{c}\right) \oplus A_{(2)}^{1,1}\left(M, a d P_{C}\right)$,

(iii) $F^{j} A_{(2)}^{\cdot}\left(M, a d P_{c}\right)=0, j \geq 2$. 
The following theorem is a consequence of $[19$, Section 7$]$, see also [4, Appendix D].

Theorem 7.2. The complex

$$
\left(A_{(2)}^{\circ}(M, a d P), A_{(2)}^{\circ}\left(M, a d P_{C}\right), F^{\circ}\right)
$$

is a Hodge complex.

As a consequence of Theorem 6.1 and 7.2 the cohomology groups of $B^{\circ}\left(M, U ; a d P_{C}\right)$ admit a Hodge structure. It is clear that

$$
H^{0}\left(B^{\circ}\left(M, U ; a d P_{C}\right)\right)=H^{1,1}\left(B^{\circ}\left(M, U ; a d P_{C}\right)\right)=C
$$

In order to understand the Hodge structure on $H^{1}\left(B^{\circ}\left(M, U ; a d P_{C}\right)\right)$ we observe that it is the direct sum of the Hodge structures on $H^{1}\left(B^{\circ}(M, U ; L)\right)$ and $H^{1}\left(B^{\circ}\left(M, U ; L^{\vee}\right)\right)$. Thus we have a direct sum decomposition

$$
\begin{gathered}
H^{1}\left(B^{\circ}\left(M, U ; a d P_{C}\right)\right)=H^{1.0}\left(B^{\circ}(M, U ; L)\right) \oplus H^{1,0}\left(B^{\circ}\left(M, U ; L^{\vee}\right)\right) \oplus \\
H^{0,1}\left(B^{\circ}(M, U ; L)\right) \oplus H^{1,0}\left(B^{\circ}\left(M, U ; L^{\vee}\right)\right)
\end{gathered}
$$

Remark 7.3. The trivial local system $E_{0}$ has no cuspidal cohomology of degree 1, i.e. $H^{1}\left(B^{\circ}\left(M, U ; E_{0}\right)\right) \doteq\{0\}$. We will abbreviate $H^{p, q}\left(B^{\circ}(M, U ; L)\right)$ (resp. $\left.H^{p, q}\left(B^{\circ}\left(M, U ; L^{\vee}\right)\right)\right)$ to $\left.H_{(2)}^{p, q}(M, L)\right)\left(\right.$ resp. $\left.H_{(2)}^{p, q}\left(M, L^{\vee}\right)\right)$.

We will now compute the dimensions of the four summands in the above Hodge decomposition. We have decomposed the complexified local system

$$
a d P_{C}=E_{0} \otimes C \oplus L \oplus L^{\vee}
$$

Recall that $E_{0}$ is a trivial local system, $L$ is the one-dimensional unitary local system with monodromy representation $\rho$ given by

$$
\rho\left(\gamma_{j}\right)=\exp \left(i 2 \theta_{j}\right), \quad 1 \leq j \leq n
$$

and $L^{\vee}$ is the dual of $L$. We note that the equation $\theta_{j}=\varepsilon_{j} r_{j}, 1 \leq j \leq n$, of $\S 3$ holds. Let $\mathbb{L}$ be the holomorphic line bundle over $M$ corresponding to $L$ and $\mathscr{L}$ be the sheaf associated to $\mathbb{L}$. We next define $\mu_{j}$ by $\mu_{j}=\theta_{j} / \pi$, whence $-1<\mu_{j}<1$. Then 


$$
\sum_{j=1}^{n} \mu_{j}=2 w \quad \text { and } \quad \sum_{j=1}^{n} \theta_{j}=2 \pi w
$$

We now define the canonical extension $\mathscr{L}_{\text {can }}$ of $\mathscr{L}$ to a holomorphic line bundle on $C \boldsymbol{P}^{1} \supset \boldsymbol{M}$. We let $\mathcal{O}$ denote the sheaf of germs of holomorphic functions on $C \boldsymbol{P}^{1}$. We define a sheaf $\mathscr{L}_{\text {can }}$ of $\mathcal{O}$-modules by

$$
\mathscr{L}_{\text {can }}(U)= \begin{cases}\Gamma(U, L), & \text { if } U \subset M \\ \Gamma_{2}(U \cap M, L), & \text { otherwise }\end{cases}
$$

Here $\Gamma_{2}$ denotes the space of square integrable holomorphic sections of $\boldsymbol{L}$ over $U \cap M$. In what follows we use $\mathscr{K}$ to denote the canonical sheaf of $\boldsymbol{C P}^{1}$.

Lemma 7.4. The sheaf $\mathscr{L}_{\text {can }}$ is locally free and $\operatorname{deg}\left(\mathscr{L}_{\text {can }}\right)=-b-2 w$.

Proof. Let $\sigma$ be the canonical multi-valued flat section of $L$. We shall denote by the same letter a lift of $\sigma$ to the universal cover of $\tilde{M}$ of $M$. We may identify the total space of $L$ with the quotient

$$
\tilde{M} \times_{\pi_{1}(M)} C
$$

and let $[\tilde{m}, x]$ denote the equivalence class of $(\tilde{m}, x)$, where $\tilde{m} \in \tilde{M}, x \in C$. Then

$$
\sigma(\tilde{m})=[\tilde{m}, 1]
$$

We see that

$$
\sigma\left(\gamma_{j} \tilde{m}\right)=\left[\gamma_{j} \tilde{m}, 1\right]=\left[\tilde{m}, \rho\left(\gamma_{j}^{-1}\right) 1\right]=\exp \left(-i 2 \theta_{j}\right) \sigma(\tilde{m})
$$

Now let $m_{j}$ be a puncture (i.e. an element of $\boldsymbol{C} \boldsymbol{P}^{1}-M$ ) and $U_{j}$ be a neighborhood of $m_{j}$ on $\boldsymbol{C P}^{1}$ (as in Section 5). Let $z$ be a holomorphic coordinate vanishing at $m_{j}$. We define $s_{j} \in \mathscr{L}_{\text {can }}(U \cap M)$ by

$$
s_{j}(z)= \begin{cases}z^{\mu_{j}} \sigma_{j}(z), & \text { if } 0<\mu_{j}<1 \\ z^{1+\mu_{j}} \sigma_{j}(z), & \text { if }-1<\mu_{j}<0 .\end{cases}
$$

Note that $s_{j}$ is a single-valued nowhere vanishing holomorphic section of $\boldsymbol{L}$ over $U \cap M$. Now suppose $s \in \mathscr{L}_{\text {can }}(U)$. We may write $s=f s_{j}$ with $f \in \mathcal{O}(U \cap M)$. We have $|s|^{2}=|f|^{2} r^{2 \mu_{j}}$. We recall that the volume element for the Poincare metric on $M$ is 


$$
\frac{r d r d \theta}{r^{2} \log ^{2} r}
$$

thus $s \in \mathscr{L}_{\text {can }}$ if and only if $f$ is regular at $m_{j}$. Thus $\mathscr{L}_{\text {can }}$ is locally free and it remains to compute its degree.

We identify $\boldsymbol{C} \boldsymbol{P}^{1}$ with $C \cup \infty$ in such a way that $m_{1}, \cdots, m_{n}$ are identified with the finite complex numbers $z_{1}, \cdots, z_{n}$. Define $\eta_{j}=1$ if $\mu_{j}<0$ and $\eta_{j}=0$ if $\mu_{j}>0$. Then

$$
s(z)=\prod_{j=1}^{n}\left(z-z_{j}\right)^{\mu_{j}+\eta_{j}} \sigma
$$

is a global meromorphic section of $\mathscr{L}_{\text {can }}$ which has no zeros in $C$. Clearly $s$ has a pole of order $\sum_{j=1}^{n}\left(\eta_{j}+\mu_{j}\right)$ at $\infty$. Since $\eta_{1}+\cdots+\eta_{n}=b$ and $\mu_{1}+\cdots+\mu_{n}=2 w$, the lemma is proved.

The proof of the next lemma is analogous to that of Lemma 7.4. We let $\left(\mathscr{L}^{\vee}\right)_{\text {can }}$ be the sheaf constructed as above using the flat line bundle $L^{v}$ on $M$ dual to $L$ (instead of $L$ ).

Lemma 7.5. The sheaf $\left(\mathscr{L}^{\vee}\right)_{\text {can }}$ is locally free and $\operatorname{deg}\left(\left(\mathscr{L}^{\vee}\right)_{\text {can }}\right)=-f+2 w$.

We can now compute the Hodge pieces $H_{(2)}^{p, q}(M, L)$ and $H_{(2)}^{p, q}\left(M, L^{\vee}\right)$. Following [4, Appendix D], we define a graded sheaf $\widetilde{\Omega}^{\bullet}$ by

$$
\tilde{\Omega}^{0}=\mathcal{O}, \quad \widetilde{\Omega}^{1}=\Omega(D)
$$

here $D=\sum_{j=1}^{n} m_{j}$, so $\operatorname{deg} D=n$. Then by [4, Proposition D.4], we have

(i) $H_{(2)}^{p, q}(M, L)=H^{q}\left(C \boldsymbol{P}^{1}, \widetilde{\Omega}^{p} \otimes \mathscr{L}_{\text {can }}\right)$;

(ii) $H_{(2)}^{p, q}\left(M, L^{\vee}\right)=H^{q}\left(C P^{1}, \widetilde{\Omega}^{p} \otimes\left(\mathscr{L}^{\vee}\right)_{c a n}\right)$;

The following theorem gives the dimensions of the Hodge summands.

Theorem 7.6. (i) $\operatorname{dim} H_{(2)}^{1,0}(M, L)=f-2 w-1$,

(ii) $\operatorname{dim} H_{(2)}^{0,1}(M, L)=b+2 w-1$,

(iii) $\operatorname{dim} H_{(2)}^{1,0}\left(M, L^{\vee}\right)=b+2 w-1$,

(iv) $\operatorname{dim} H_{(2)}^{0,1}\left(M, L^{\vee}\right)=f-2 w-1$,

Proof. Use the previous formulas for the Hodge structure and the 
Riemann-Roch theorem for $\boldsymbol{C P}^{1}$.

We now extend the quadratic form $Q$ of Section 5 to a Hermitian form $H$ on the complexified first cohomology. Recall that $Q$ is induced by the pointwise bilinear form $b=b_{1} \otimes b_{2}$ on $T^{*}(M) \otimes a d P$. We extend $b_{1}, b_{2}$ to a vector-valued skew-hermitian forms $h_{1}, h_{2}$ on $T^{*}(M) \otimes C$ and $a d P \otimes C$ respectively by the following formulas

$$
\alpha \wedge \bar{\beta}=h_{1}(\alpha, \beta), \quad[u, \bar{v}]=h_{2}(u, v)
$$

Here $\alpha, \beta \in T^{*}(M) \otimes C$ and $u, v \in a d P_{C}$. Note that the standard fiber of $L$ is spanned by $\lambda=e_{1}-i e_{2}$ and the standard fiber of $L^{\vee}$ is spanned by $\bar{\lambda}=e_{1}+i e_{2}$. Then $[\lambda, \bar{\lambda}]=2 i e_{3}$. Thus $h_{1}$ and $\left.h_{2}\right|_{E \otimes C}$ take values in trivial line bundles over $M$. We identify these forms with scalar-valued Hermitian forms using the bases $v o l$ for $\Lambda^{2} T^{*}(M)$ and $e_{3}$ for $E_{0}$. The following lemma is immediate.

Lemma 7.7. (i) The subspaces $\left(T^{*}\right)^{1,0}(M)$ and $\left(T^{*}\right)^{0,1}(M)$ are orthogonal for $h_{1}$.

(ii) The form $i h_{1}$ is positive definite on $\left(T^{*}\right)^{1,0}(M)$ and negative definite on $\left(T^{*}\right)^{0,1}(M)$.

(iii) The subspaces $L$ and $L^{\vee}$ are orthogonal for $h_{2}$.

(iv) The form - ih $h_{2}$ is positive definite on $L$ and negative definite on $L^{v}$.

Corollary 7.8. The tensor product $h=h_{1} \otimes h_{2}$ is positive definite on $\left(T^{*}\right)^{1,0}(M) \otimes L$ and $\left(T^{*}\right)^{0,1}(M) \otimes L^{\vee}$ and negative definite on $\left(T^{*}\right)^{0,1}(M) \otimes L$ and $\left(T^{*}\right)^{1,0}(M) \otimes L^{\vee}$.

We now define a hermitian form $H$ on $H_{(2)}^{1}\left(M, a d P_{c}\right)$ by

$$
H(\eta, \zeta)=\int_{M} h(\eta, \zeta)
$$

The pointwise results of Lemma 7.7 imply the following lemma.

Lemma 7.9. (Hodge-Riemann bilinear relations)

(i) The four Hodge summands are orthogonal for $H$.

(ii) The restricion of $H$ to each of the four summands is definite, $H$ is 
positive definite on the two summands of dimension $f-2 w-1$ and negative definite on the two summands of dimension $b+2 w-1$.

Corollary 7.10. The form $H$ is nonsingular of signature $(2(f-2 w-1)$, $2(b+2 w-1))$.

We next compute the signature of the restriction of the form $H$ to the subspace

$$
H_{(2)}^{1}\left(M, a d P_{C}\right)^{Z / 2}
$$

of $Z / 2$-invariants. Recall that the $Z / 2$ action is induced by the action of $\tau$ on $M$ and the action of $\rho_{0}(\tau)$ on the coefficients of the (complexified) monodromy action. We recall that we have chosen

$$
\rho_{0}(\tau)=\rho_{0}\left(\tau_{1}\right)=\left(\begin{array}{ccc}
1 & 0 & 0 \\
0 & -1 & 0 \\
0 & 0 & -1
\end{array}\right)
$$

We find that the action of $\tau$ on coefficients of $E=L \oplus L^{\vee}$ is given by

$$
\rho(\tau) \lambda=-\bar{\lambda}, \quad \rho(\tau) \bar{\lambda}=-\lambda
$$

Thus $\rho(\tau)$ realizes the duality on $H_{(2)}^{1}\left(M, a d P_{C}\right)$, it interchanges the two $(f-2 w-1)$-dimensional components and the two $(b+2 w-1)$-dimensional components. Thus,

$$
H_{(2)}^{1}\left(M, a d P_{C}\right)^{Z / 2}=\left(H_{(2)}^{1,0}(M, L) \oplus H_{(2)}^{0,1}\left(M, L^{\vee}\right)\right)^{Z / 2} \oplus\left(H_{(2)}^{1.0}\left(M, L^{\vee}\right) \oplus H_{(2)}^{0,1}(M, L)\right)^{Z / 2}
$$

We obtain the following lemma.

Lemma 7.11. The induced hermitian form on

$$
H_{(2)}^{1}\left(M, a d P_{C}\right)^{Z / 2}
$$

has signature $(f-2 w-1, b+2 w-1)$.

We now restrict to the real subspace $H_{(2)}^{1}(M, a d P)$ of $H_{(2)}^{1}\left(M, a d P_{C}\right)$ and obtain the following theorem. 
Theorem 7.12. The quadratic form $Q$ on $H_{(2)}^{1}(M, a d P)^{\mathbf{Z} / 2}$ has signature $(f-2 w-1, b+2 w-1)$.

Proof. We will prove that the above splitting of $H_{(2)}^{1}\left(M, a d P_{c}\right)^{Z / 2}$ is defined over $\boldsymbol{R}$. Indeed, forms in the first summand may be represented by $f(d z \otimes \lambda+d \bar{z} \otimes \bar{\lambda})$ and forms of the second summand by $g(d z \otimes \bar{\lambda}+d \bar{z} \otimes \lambda)$ (recall that we identify $M$ with $C \cup\{\infty\}-\left\{z_{1}, \cdots, z_{n}\right\}$. Both spaces of forms are clearly defined over $\boldsymbol{R}$.

Corollary 7.13. The quadratic form $Q$ on $H^{1}\left(B^{\circ}(M, U ; \text { adP })\right)^{Z / 2}$ has signature $(f-2 w-1, b+2 w-1)$.

Let $Z(Q)$ be the null-cone $\{Q=0\} \subset H^{1}\left(B^{\circ}(M, U ; a d P)\right)^{Z / 2}$.

We can now prove Theorem 1.1 of the Introduction. We claim

$$
H^{0}\left(B^{*}(M, U ; a d P)\right)^{\mathbf{Z} / 2}=\{0\}
$$

Indeed, $H^{0}\left(B^{\circ}(M, U ; a d P)\right) \cong R e_{3} \subset s o(3)$ and the action of $Z / 2$ on this space is easily seen to be given by multiplication by -1 . By Theorem 5.2 , the augmentation ideal

$$
\left(B^{*}(M, U ; a d P)\right)_{0}^{\mathbf{Z} / 2}
$$

is a controlling differential graded Lie algebra for the deformations of $\Pi_{0}$. We now apply Theorem 3.5 of [8] noting the cone $\mathscr{2}_{H(L)}$ of that theorem is the cone $Z(Q)$ discussed in the above corollary.

Theorem 1.1 is now immediate from Theorem 5.4, Corollary 6.9 and Corollary 7.13 .

It is immediate from the above that a spherical polygonal linkage is (locally) rigid if and only if the quadratic form $Q$ is definite, which is equivalent to: $f-2 w-1=0$ or $b+2 w-1=0$. We thus obtain Corollary 1.2 from the Introduction.

\section{§8. The Topology of the Moduli Space of a Spherical Polygonal Linkage}

In this section we combine our results in $\$ 7$ with results of $A$. Galitzer [6] to give a "wall-crossing" algorithm for computing the topology of the moduli spaces $M_{\mathrm{r}}$.

Let $\mathscr{P}_{n}$ denote the space of all $n$-gons $\sigma=\left(\sigma_{1}, \cdots, \sigma_{n}\right)$ in $S^{2}$ (see the 
definition in $\S 3)$. Thus $\mathscr{P}_{n} \cong\left(S^{2}\right)^{n}$. Let $\mathscr{Q}_{n}$ denote the quotient of $\mathscr{P}_{n}$ by $S O(3)$ and let $\pi: \mathscr{Q}_{n} \rightarrow \boldsymbol{R}^{n}$ be the map given by $\pi(\sigma)=r=\left(r_{1}, \cdots, r_{n}\right)$, where $r_{i}$ is the length of the geodesic arc $\sigma_{i}, 1 \leq i \leq n$. We let $D_{n}\left(S^{2}\right)$ denote the image of $\pi$. In [6] A. Galitzer has described $D_{n}\left(S^{2}\right)$. We will need some notation to describe her results. If $I \subset\{1,2, \cdots, n\}$ we let $\bar{I}$ denote the complement of $I$, $|I|$ be the cardinality of $I$ and $r_{I}=\Sigma_{i \in I} r_{i}$. Define a polyhedron $K_{n} \subset \mathbb{R}^{n}$ by the system of inequalities

$$
\begin{aligned}
& 0 \leq r_{i} \leq \pi, \quad 1 \leq i \leq n, \quad \text { and } \\
& r_{I} \leq r_{\bar{I}}+(|I|-1) \pi, \quad I \subset\{1,2, \cdots, n\}, \quad \text { with }|I| \text { odd }
\end{aligned}
$$

Then Galitzer proves

Theorem 8.1. $K_{n}=D_{n}\left(S^{2}\right)$.

In addition she proves that the codimension 1 faces of $D_{n}\left(\mathbb{S}^{2}\right)$ are given by the intersections of the hyperplanes corresponding to the above inequalities with $K_{n}$, i.e. the above representation of $K_{n}$ is irredundant.

The space $\mathscr{Q}_{n}$ is difficult to work with since it has singularities corresponding to fixed points of subgroups of $S O(3)$. To remedy this we let $\mathscr{P}_{n}^{0}$ denote the open subset of $\mathscr{P}_{n}$ corresponding to those $n$-gons such that successive vertices do not coincide and are not antipodal. We let $\mathscr{2}_{n}^{0}$ denote the quotient of $\mathscr{P}_{n}^{0}$ by $S O(3)$. Then $\mathscr{Q}_{n}^{0}$ is naturally a smooth manifold of dimension $2 n-3$. Indeed, $\mathscr{2}_{n}^{0}$ is naturally diffeomorphic to the submanifold $S \subset \mathscr{P}_{n}^{0}$ consisting of those $n$-gons with the vertex set $u=\left(u_{1}, \cdots, u_{n}\right)$ satisfying

$$
u_{1}=\overrightarrow{e_{1}}, \quad\left(u_{2}, \overrightarrow{e_{3}}\right)=0, \quad\left(u_{2}, \overrightarrow{e_{2}}\right)>0
$$

Note that $\pi\left(\mathscr{Q}_{n}^{0}\right) \supset \operatorname{int}\left(K_{n}\right)=K_{n}^{0}$. We will henceforth replace $\pi$ by its restriction to $\mathscr{Q}_{n}^{0}$.

We shall see shortly that the set of critical values of $\pi$ inside $K_{n}^{0}$ is the union of a collection of hyperplane sections of $K_{n}^{0}$. We call these hyperplane sections walls of $K_{n}$. Connected components in $K_{n}^{0}$ of the union of walls are called chambers. In [6] Galitzer determines the walls of $K_{n}$. We again summarize her results.

Let $I \subset\{1, \cdots, n\}$ be any non-empty subset. For each nonnegative integer $w$ let $H_{I, w}$ denote the hyperplane in $\boldsymbol{R}^{n}$ defined by the equation 


$$
r_{I}-r_{\bar{I}}=2 \pi w
$$

We then have the following lemma of Galitzer

Lemma 8.2. $\quad H_{I, w} \cap K_{n}^{0} \neq \emptyset \Leftrightarrow|I| \geq 2 w+2$.

We now prove that the hyperplanes $H_{I, w}$ with $|I| \geq 2 w+2$ are the walls of $K_{n}$.

Lemma 8.3. Let $r \in K_{n}^{0}$. Then $r$ is not a regular value of $\pi$ if and only if $r \in H_{I, w}$ for some $I, w \geq 0$ with $|I| \geq 2 w+2$.

Proof. Suppose first that $r$ is on $H_{I, w}$ with $|I| \geq 2 w+2$. We will show that $\pi^{-1}(r)$ is singular, which would mean that $r$ is not a regular value of $\pi$. Since $r$ is on a wall there is a subset $I \subset\{1, \cdots, n\}$ such that $r_{I}-r_{\bar{I}}=2 \pi w$ with $|I| \geq 2 w+2$. We can construct a degenerate $n$-gon $u$ in $\pi^{-1}(r)$ by taking $\bar{I}$ to be the set of indices corresponding to the back-tracks and $I$ to be the set of indices corresponding to forward-tracks. (Note: we do not assume here that $u$ belongs to the cross-section $S_{r}$.) Since $r_{I}-r_{\bar{I}}=2 \pi w$ the resulting degenerate linkage closes up. By Theorem 7.12 we find that $u$ is a singular point on $M_{r}$ because the germ of $M_{r}$ at $u$ is isomorphic to the germ of a quadratic cone of signature $(f-2 w-1, b+2 w-1)$ at 0 . Thus $\pi^{-1}(r)$ is singular.

Conversely, suppose that $r$ is not on a wall of $K_{n}$. This implies that $M_{r}$ contains no degenerate polygons. Let $u \in \pi^{-1}(r)$. Now the kernel of $d \pi: T_{u}\left(\mathscr{Q}_{n}^{0}\right)$ $\rightarrow T_{r}\left(\boldsymbol{R}^{n}\right)$ is the Zariski tangent space $T_{u}\left(M_{r}\right)$ of $M_{r}$ at $u$. By Theorem 7.12 we have

$$
T_{u}\left(M_{r}\right) \cong H^{1}\left(B^{*}(M, U ; a d P)^{\mathbf{Z} / 2}\right)
$$

Since $u$ is nondegenerate the corresponding representation $\rho$ is irreducible and

$$
\operatorname{dim} H^{1}\left(B^{\bullet}(M, U ; a d P)^{\mathbf{Z} / 2}\right)=n-3
$$

Hence $\operatorname{dim} \operatorname{Im}\left(d \pi_{u}\right)=(2 n-3)-(n-3)=n$ and $d \pi_{u}$ is onto.

Since $\pi$ is proper it is a fibration over each chamber and the topology of the fibers does not change within a chamber. We now compute how the topology of the fibers changes when we cross a wall.

Suppose that $r^{*} \in K_{n}^{0}$ lies on the intersection of the walls

$$
H_{I_{1}, w_{1}}, H_{I_{2}, w_{2}}, \cdots, H_{I_{p}, w_{p}}
$$


Choose $u^{*}$ a degenerate linkage with $\pi\left(u^{*}\right)=r^{*}$. Let $L \subset K_{n}^{0}$ be the line segment defined by $r_{i}=r_{i}^{*}, 1 \leq i \leq n-1$ and $-\varepsilon \leq\left|r_{n}-r_{n}^{*}\right| \leq \varepsilon$. Here $\varepsilon$ is chosen so that $L$ doesn't intersect any wall except at $r^{*}$. Let $X_{L}=\pi^{-1}(L)$.

Lemma 8.4. $X_{L}$ is a smooth submanifold of $\mathscr{P}_{n}$ of dimension $n-2 . \quad$ The inclusion $i: M_{r *} \subseteq X_{L}$ induces an isomorphism of tangent spaces

$$
i_{*}: T_{u^{*}}\left(M_{r^{*}}\right) \rightarrow T_{u^{*}}\left(X_{L}\right)
$$

Proof. A point in $X_{L}$ is a closed $n$-gon where the lengths of the first $n-1$ sides are prescribed to be $r_{1}^{*}, r_{2}^{*}, \cdots, r_{n-1}^{*}$ but the length of the $n$-th side is not determined. The operation of forgetting the $n$-th side gives an isomorphism to the moduli space of the free linkage where the underlying map is obtained by deleting the $n$-th side of the $n$-gon. Clearly the moduli space of such a free linkage is the product of $n-2$ circles.

Since $\operatorname{dim}\left(T_{u^{*}}\left(M_{r^{*}}\right)\right)=n-2, \operatorname{dim}\left(T_{u^{*}}\left(X_{L}\right)\right)=n-2$, and $i_{*}$ is an injection, it is necessarily an isomorphism.

Remark 8.5. In the above $T_{u^{*}}\left(M_{r^{*}}\right)$ is the Zariski tangent space of $M_{r *}$. Note that

$$
\operatorname{dim} T_{u^{*}}\left(M_{r^{*}}\right)=\operatorname{dim} M_{r^{*}}+1
$$

Every infinitesimal deformation of $u^{*}$ in $M_{r^{*}}$ is tangent to a curve in $X_{L}$.

Thus we have reduced the problem of finding a wall-crossing formula to computing how the level sets of $r_{n} \mid X_{L}$ change when we pass from $r_{n}^{*}-\varepsilon$ to $r_{n}^{*}+\varepsilon$. Our desired formula will be a consequence of the next three lemmas.

Lemma 8.6. If $u \in X_{L}$ is a critical point of $r_{n} \mid X_{L}$ then $u$ is degenerate.

Proof. Our arguments essentially repeat the proof of Lemma 8.3. Let $u \in X_{L}$ and $V=\operatorname{ker} d\left(r_{n} \mid X_{L}\right)_{u}$. Then $V \subset T_{u}\left(X_{L}\right)$. By definition $V$ is the Zariski tangent space of $u$ to the fiber of $r_{n} \mid X_{L}$ through $u$. Hence

$$
V \cong H^{1}\left(B^{\circ}(M, U ; a d P)^{Z / 2}\right)
$$

Therefore if $u$ is nondegenerate then $\operatorname{dim}(V)=n-3$ and

$$
\operatorname{dim}\left(\operatorname{Im} d\left(r_{n} \mid X_{L}\right)_{u}\right)=1
$$


Lemma 8.7. $r_{n} \mid X_{L}$ has exactly $p$ critical points.

Proof. We apply Lemma 8.6 and observe that any critical point of $r_{n} \mid X_{L}$ must be a degenerate linkage $v$ lying in $\pi^{-1}\left(r^{*}\right)$. Let $J$ be the set of forward-tracks and $w$ be the winding number of $v$. Then $r_{J}-r_{\bar{J}}=2 \pi w$. Hence there exists $i$ between 1 and $p$ such that $J=I_{i}, w=w_{i}$. But a degenerate linkage in $M_{r^{*}}$ is determined by its set of forward-tracks.

Let $u_{1}^{*}, u_{2}^{*}, \cdots, u_{p}^{*}$ be the set of critical points of $r_{n} \mid X_{L}$. Let $u^{*}$ be one of these critical points and let $Q_{L}^{*}$ be the Hessian of $r_{n} \mid X_{L}$ at $u^{*}$. Recall that

$$
Q: H^{1}\left(B^{*}(M, U ; a d P)^{\mathbf{Z} / 2}\right) \rightarrow \boldsymbol{R}
$$

denotes the cup-product. By Theorem 5.4 we have a commutative diagram (with the horizontal arrows $\phi$ and $\psi$ isomorphisms and the vertical arrows $\eta$ and $\eta^{\prime}$ the canonical projections):

$$
\begin{array}{ccc}
T_{u^{*}}\left(M_{r^{*}}\right) \stackrel{\phi}{\rightarrow} & H^{1}\left(B^{*}(M, U ; a d P)^{\mathbf{Z} / 2}\right) \\
{ }^{\eta} \uparrow & & \uparrow^{\eta^{\prime}} \\
T_{u^{*}}^{(2)}\left(M_{r^{*}}\right) \stackrel{\psi}{\rightarrow} & T_{\rho_{0}}^{(2)}(\operatorname{Hom}(\Phi, R ; S O(3)) / S O(3)
\end{array}
$$

Here $T^{(2)}$ denotes the 2-jet bundle, i.e. the equivalence classes of formal analytic curves up to order three contact (we recall that $M_{r}$ has at worst quardratic singularities). The image of $\eta^{\prime}$ is the null-cone $Z(Q)$ of $Q$ by Theorem 5.4, since the cup-product $Q$ is the obstruction to lifting a tangent vector to a 2 -jet, see $[8, \S 4.4]$.

Combining the isomorphism $\phi$ above with the isomorphism $i_{*}$ of Lemma 8.6 we obtain a canonical isomorphism

$$
\tau: T_{u^{*}}\left(X_{L}\right) \cong H^{1}\left(B^{*}(M, U ; a d P)^{Z / 2}\right)
$$

The following Lemma gives the critical link between Morse theory and deformation theory.

Lemma 8.8. Under the isomorphism $\tau$ the null cone $Z\left(Q_{\mathrm{L}}^{*}\right)$ of $Q_{\mathrm{L}}^{*}$ is carried onto the null-cone $Z(Q)$ of $Q$.

Proof. Suppose that $\alpha \in T_{u^{*}}\left(X_{L}\right)$ is annihilated by $Q_{L}^{*}$. Let $a(t)$ be a curve 
in $X_{L}$ such that $a(0)=u^{*}$ and $a^{\prime}(0)=\alpha$. Then $r_{i}(a(t))=r_{i}^{*}, 1 \leq i \leq n-1$ and $r_{n}(a(t)) \equiv r_{n}^{*}\left(\bmod t^{3}\right)$. Thus $a(t)$ induces a 2-nd order deformation of the linkage $u^{*}$ in $M_{r^{*}}$, i.e. an element $\delta \in T^{(2)}\left(M_{r^{*}}\right)$ such that $\eta(\delta)=\alpha$. But by [13], Theorem 3.2 , the image of $\delta$ under $\psi$ is a 2-nd order deformation $\gamma$ of the representation $\rho^{*}$ corresponding to $u^{*}$. Since $\eta^{\prime}(\gamma)=\phi(\alpha)$ we have $\phi\left(Z\left(Q_{L}^{*}\right)\right) \subset Z(Q)$. Conversely, let $\beta \in Z(Q)$. Choose $\gamma \in\left(\eta^{\prime}\right)^{-1}(\beta)$ and put $\delta=\psi^{-1}(\gamma)$. Since $X_{L}$ is smooth and $T^{(2)}\left(M_{r^{*}}\right) \subset T^{(2)}\left(X_{L}\right)$, the 2-jet $\delta$ is represented by a curve $a(t)$ in $X_{L}$ preserving $r_{n}$ up to term of order 3. Let $\alpha=\eta(\delta)$. Then $\alpha \in Z\left(Q_{L}^{*}\right)$. But $\alpha=\phi^{-1}(\beta)$.

\section{Corollary 8.9. $Q_{L}^{*}$ is nondegenerate.}

Proof. We have seen that $Q$ is nondegenerate. Hence the projectivization of $Z(Q)$ is smooth. Hence the projectivization of $Z\left(Q_{L}^{*}\right)$ is smooth. But a quadratic form is nondegenerate if and only if the projectivization of its null cone is smooth. Hence $Q_{L}^{*}$ is nondegenerate.

Since Corollary 7.13 determines $Z(Q)$ and $Z(Q)=Z\left(Q_{L}^{*}\right)$ we obtain

Theorem 8.10. $\quad r_{n} \mid X_{L}$ is a Morse function with a finite collection of critical points $u_{1}^{*}, \cdots, u_{p}^{*}$, all located on the critical fiber $M_{r^{*}}$. The critical point $u_{i}^{*}$ corresponds to a degenerate n-gon linkage in $M_{r *}$ with $f_{i}$ forward-tracks, $b_{i}$ back-tracks and winding number $w_{i}$. Then the signature of the Hessian of $r_{n} \mid X_{L}$ at $u_{i}^{*}$ is either $\left(f_{i}-2 w_{i}-1, b_{i}+2 w_{i}-1\right)$ or $\left(b_{i}+2 w_{i}-1, f_{i}-2 w_{i}-1\right)$ depending on the orientation of the great circle containing $u_{i}^{*}$.

\section{References}

[ 1 ] Boden, H. and Hu, Y., Variations of moduli of parabolic bundles, Math. Ann., 301 (1995), 539-559.

[ 2 ] Boden, H. and Yokogawa, K., Moduli spaces of parabolic Higgs bundles and parabolic $K(D)$ pairs overs smooth curves: I, Preprint.

[ 3 ] Deligne, P., Griffiths, P., Morgan, J. and Sullivan, D., Rational homotopy type of compact Kahler manifolds, Invent. Math., 29 (1975), 245-274.

[ 4 ] Esnault, H. and Viehweg, E., Logarithmic de Rham complexes and vanishing theorems, Invent. Math., 86 (1986), 161-194.

[ 5 ] Fintushel, R. and Stern, E., Instanton homology of Seifert fibered homology three spheres, Proc. of London Math. Soc., (3) 61 (1990), 109-137.

[6] Galitzer, A., Ph.D. Thesis, University of Maryland, 1996.

[ 7 ] Godement, R., Theorie des fasceaux, Hermann, 1973.

[ 8 ] Goldman, W. and Millson, J.J., The deformation theory of representations of fundamental 
groups of compact Kahler manifolds, IHES, Publ. Math., 67 (1988), 43-96.

[ 9 ] Goldman, W. and Millson, J.J., The homotopy invariance of the Kuranishi space, Illinois J. Math., 34 (1990), 337-367.

[10] Kapovich, M. and Millson, J.J., On the moduli space of polygons in the Euclidean plane, J. Diff. Geom., 42 (1995) N 1, 133-164.

[11] - The symplectic geometry of polygons in Euclidean space, J. Diff. Geom., 44 (1996), 479-513.

[12] - On the deformation theory of representations of fundamental groups of hyperbolic 3-manifolds, Topology, 35 (1996) N4, 1085-1106.

[13] - The relative deformation theory of representations and flat connections and deformations of linkages in constant curvature spaces, Compositio Math., 103 (1996), 287-317.

[14] - On representation varieties of Artin groups, projective arrangements and the fundamental groups of smooth complex algebraic varieties, Preprint.

[15] Kirk, P. and Klassen, E., Representation spaces of Seifert fibered homology spheres, Topology, 30 (1991), 77-95.

[16] Mehta, V. and Seshadri, C., Moduli of vector bundles on curves with parabolic structures, Math. Ann., 248 (1980), 205-239.

[17] Millson, J. J., Rational homotopy theory and deformation problems from algebraic geometry, Proceedings of ICM 1990, I, 549-558.

[18] Simpson, C., Harmonic bundles on noncompact curves, J. AMS, 3 (1990), 713-770.

[19] Zucker, S., Hodge theory with degenerating coefficients: $L_{2}$ cohomology in the Poincare metric, Ann. of Math., 109 (1979), 415-476. 
